\title{
The UPIC system as an instrument of learning
}

\author{
PETER NELSON \\ Faculty of Music, The University of Edinburgh, 12 Nicolson Square, Edinburgh EH8 9DF, UK \\ E-mail: P.Nelson@music.ed.ac.uk
}

The conceptual basis of the UPIC system is described and discussed, in particular its relation to the work of its orginator, Iannis Xenakis, to the science of acoustics and to the development of an intuitive graphic imagery. A possible pedagogical approach to musical creation using the resources of the UPIC is developed, and the particular pedagogical ethos of the system is emphasised. There follows a detailed description of the practical arrangements for a number of different workshop situations using the UPIC system in which the author has participated, and an evaluation of the outcomes of using the system in this way.

When Iannis Xenakis conceived the UPIC system, perhaps prompted by the ideas of Edgar Varèse (Matossian 1986: 241), it was as a solution to some of the technical problems surrounding his own compositions. Yet right from the very beginning the pedagogical possibilities of the system were important to him. His view of music has always bridged the physical and the cultural, and since he saw computers as opening up hitherto unavailable opportunities for investigating and controlling the physical world of sound, he saw no reason why they should not also provide a universal access to these opportunities (Lohner 1986b). Universal access is one of the tenets of industrial modernism and the 1960s and 1970s were a time of optimism for the new computer revolution. The social potential seemed immense; that whatever their age, economic situation or physical capabilities, everyone could have the possibility to realise their own creative and imaginative powers through the mediation of the power of the machine. A machine which would also bring people face to face with the insights of scientific investigation and the wonder of the created universe. The UPIC is conceived very much in the spirit of the computer age, but it also has a certain rough pragmatism which turns out to be a huge advantage in its interface with the user. Universality of access requires careful design, and the UPIC is a masterpiece of technological insight in combining simplicity of means with sophistication of output. The key is the capacity of the human hand to draw.

Perhaps because of his background in engineering and architecture, Xenakis' preliminary compositional work has always involved a degree of graphic design: at the very least the planning of formal shapes, processes and proportions, and, in the case of his first large-scale works, the preparation of complete graphic scores that had to be laboriously transcribed into conventional musical notation. Anecdotally, the UPIC was conceived as a way of avoiding this labour by transferring this graphism directly into sound, but two more fundamental ideas lie behind this.

First there was, of course, Xenakis' desire to create an electronic instrument more capable of realising his statistical and glissandoing sound masses than conventional instruments, and with the possibility of timbres and pitch structures free of the cultural accretions of those instruments and of the straitjacket of the theory of Fourier (Xenakis 1991: 258). But also there was the intuitive connection between those principles of sonic evolution which interested Xenakis (Xenakis 1992: 8-10) and the natural, graphic flow of the hand. The curves and sweeps of Metastasis, or indeed the arborescent patterns that lie behind many later works, are essentially flowing and transformational in a continuous way which is alien to the discrete note heads of conventional music notation and integral to the processes of evolution in all natural structures, including those of sound (Xenakis 1992: chap. 11). Thus the graphical precomposition is not an accident, or simple predilection, but goes to the heart of the musical conception: a conception which includes the way of the graphic hand in the way that instrumental performance includes the way of the dextrous hand, trained to the geography of an instrument. Here, as in much of Xenakis' thinking, temporally isolated note events become merely a special case in a more fundamentally complete theory; not denied but contextualised in a world of continuities.

The intuitive nature of the graphical domain, the direct connection between the hand and the brain, is perhaps the main link between the UPIC system and a pedagogical method. One of the problems with highly evolved symbolic systems, like musical notation, is the steep learning curve towards their mastery. Both the complexity of the graphic elements and the inherent opacity of their symbolic meanings result in a distancing of the system from normal 
experience and the imposition of special, nonanalogous structures. Furthermore, because of the particular evolution of musical notation from an essentially mnemonic role in an aural culture, it has some quite severe limitations in its relationship to the natural sound world. A minimally constrained graphism, on the other hand, is more secure in its possible relations to perceived sound and, while naive in its rudimentary imitations, is in fact capable of rapid and intuitive development into more complex situations.

It was clear to Xenakis from the start that even quite small children are capable of finding a parallel between a sound which rises and falls and a line which rises and falls, and that from this simple beginning, and the natural predisposition of humans to draw in imitation of the world, could come a fast and yet uncompromising access to the world of sound and musical composition. Hence the conception of the UPIC as an instrument where one draws music.

The technical specifications of all the various versions of the UPIC system as it came into being have been already described elsewhere in some detail (Lohner 1986a, b, Powel 1988, Marino, Razcinski and Serra 1990, 1991, 1993, Mosch 1993) so I will not go into them here. However, one or two of those details are worth emphasising, to show the clear connection between the intellectual and the corporeal which is at the heart of the UPIC. The original versions of the system had at their centre a large digitising drawing board of the kind commonly used in CAD/CAM systems. The proportions of these boards are ergonomically designed to follow the proportions of the human hand and arm in motion, making the physical traversal of the space natural and intuitive. Thus the scale of the graphic images is always easy to judge and control. The software was controlled simply by clicking the board's pen within a number of option boxes placed on the surface of the board itself, so there was no alphanumeric entry of any sort. The software commands were mostly very simple, controlling the most basic drawing and storage functions; however, more sophisticated operations such as the complete reconfiguration of the surface of the drawing board were also possible. Again this was done by hand and pen, not by numerical input, thus taking the physical proportions of the user naturally into account. Lastly, the board had a very high resolution allowing the detail of small hand movements to be captured as well as the larger gestures. Point co-ordinates, as well as frequency, loudness in $\mathrm{dB}$ and other relevant information, were displayed on a video monitor beside the board, for those who wanted to make use of them. Thus very precise drawing was possible by moving the hand over the surface of the board while watching the measurements displayed on the video monitor. The system was thus both true to the bodily and gestural proportions of the user, and responsive to the simplest or most measured of movements.

It has to be said that, while these boards are expensive, they are hugely more appropriate to the spirit and efficacy of the UPIC than later versions operated from a mouse: a very poor drawing tool.

Before I go on to discuss some of my experiences of using the UPIC in different sorts of situations and with people of different ages, backgrounds and physical capabilities, it might be useful to consider some of the crucial issues in learning to conceive music on the UPIC, and the way in which these might have wider musical significance. Although the learning process might go faster or slower, or take a slightly different path depending on who is involved, it always begins with these issues which are manifested on the UPIC in a unique way. Furthermore, they all have to do with the nature of sound rather than with the nature of music. The pedagogical starting point is physics and acoustics and the music springs from that. Of course this mirrors certain aspects of Xenakis' own aesthetic position, but it also allows a great deal of freedom and quite forces an attitude of experimentation, as will become apparent.

The first, and most important, of these issues concerns the idea of the envelope of a sound. To children, especially, this can be a rather opaque concept. The physical energy required to make a sound is apparent, either through the volition which drives the breath through the vocal chords or through the force required for mechanical action on a body. However, an electronic instrument only mimics this energy and to control it requires that it have some representation. Unlike a normal synthesizer, the UPIC begins each session as a tabula rasa. It cannot make a sound until something is drawn. Thus the user is brought immediately to the issue of the sonic envelope, and drawing turns out to be a simple and effective method of representation.

A good practical approach to this starting point is for the facilitator to use the single pre-stored waveform, a sine wave, and then to configure the table so that the output amplitude is represented on the vertical axis (the horizontal axis is unused in this instance). Now a sound of loudness corresponding to the distance of the pen from the bottom of the table is produced as the pen is pressed onto the table. As the pen is moved up and down, envelopes are drawn which obviously give a graphic representation of the perceived sound. From this beginning, an inventory of simple envelopes can be drawn and stored in the UPIC's palette for future use.

Since the video monitor displays the output in $\mathrm{dB}$, the more advanced student can note the perceptual results of different shapes with some accuracy. For instance, a conventional ADSR envelope, as drawn 


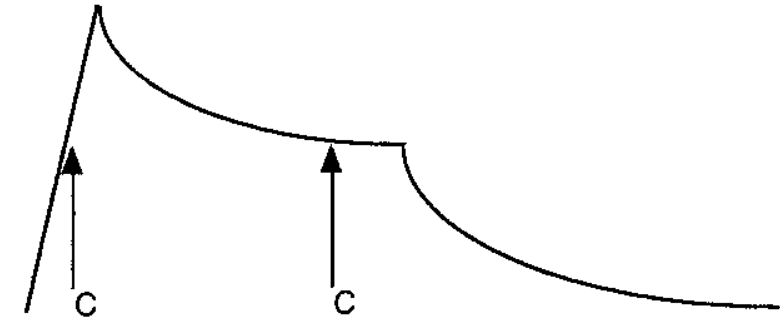

Figure 1

in figure 1, may well have a perceived cutoff at C, depending on the position of the image on the vertical axis. Thus graphic envelopes do not always represent what is heard quite so simply, and this demonstration develops a sensitivity to $\mathrm{dB}$ ranges and their relation to frequency, for example.

Furthermore, the UPIC is in fact capable of sampling sounds and extracting their envelopes (figure 2). Since envelopes can have arbitrary complexity, down to the resolution of the table, an investigation into the nature of envelopes is possible. Indeed at this point it becomes obvious that sophistication of envelope is crucial to the creation of sounds that are interesting to the ear.

The second issue, which might seem of equal importance to that of the envelope, is the issue of waveforms. Since the UPIC begins as a tabula rasa, even these must be drawn by hand, and this leads into one of the most crucial and challenging aspects of the system. Xenakis' understanding of the nature of sound and its representation owes little to Fourier and much to Einstein (Xenakis 1992: xiii). The graphic representation of a sound as a series of instantaneous amplitudes is a line of varying height on the energy (amplitude) axis, extended on the time axis. As sound approaches harmonicity and then pitch, this line approaches a cyclic waveform. Thus using the UPIC, even with quite young children, necessitates an approach to the physics of sound and the concepts of inharmonicity, harmonicity and waveform.

Practically speaking, there are various ways into this when working with young people or those who know little of physics. One could try drawing the waves of the sea, or the motion of the skin of a drum as it is hit, but neither of the resultant graphics produces a sound anywhere near the analogy. At the very least this demonstrates the crucial relationship between timbre and envelope, but it also demonstrates clearly the importance of the time-varying aspects of sound and the particular aural intransigence of static waveforms. This could be construed

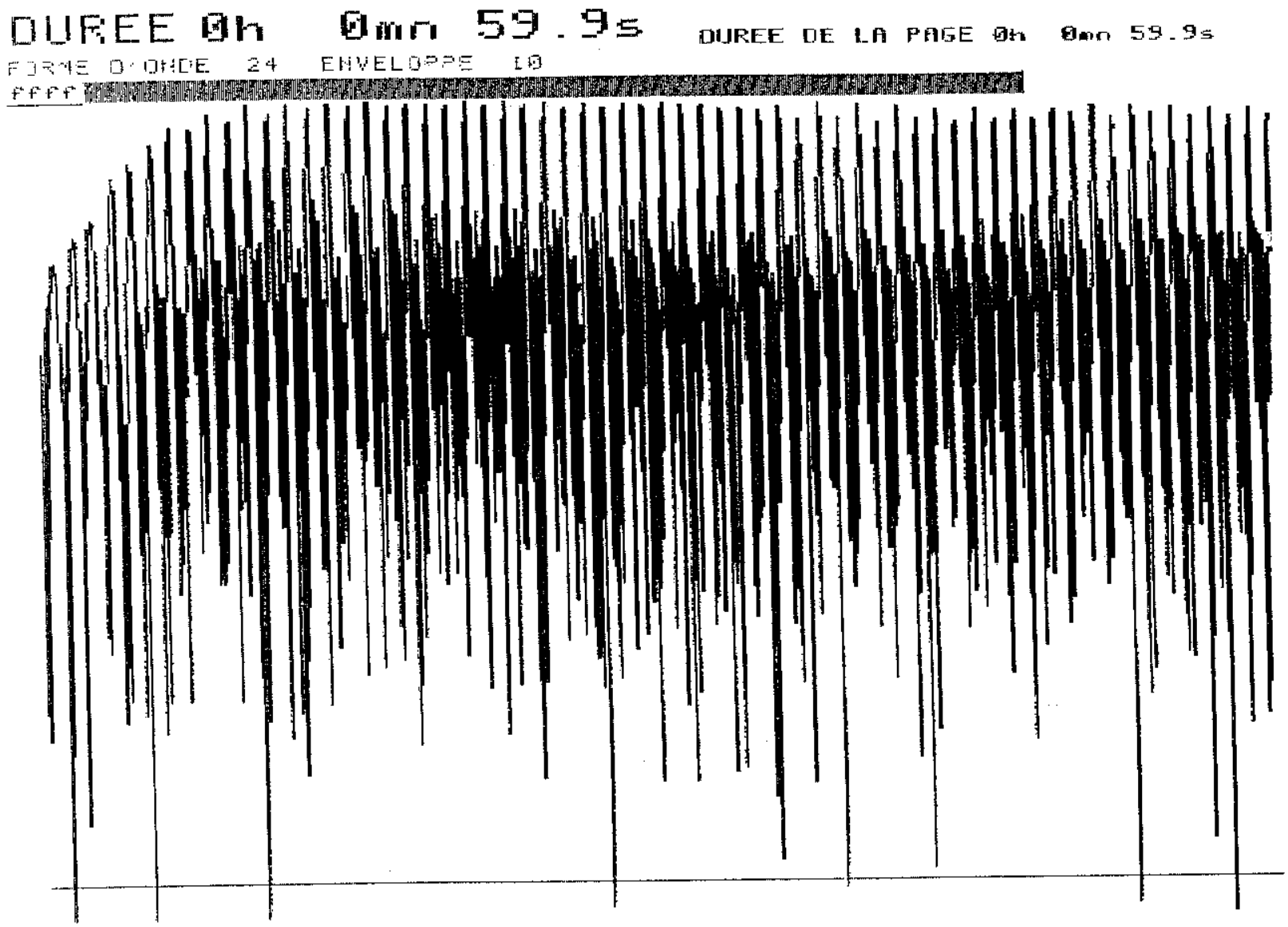

Figure 2 
as being a severe pedagogical difficulty, possibly leading to much confusion and the erection of an impermeable barrier between the imagination of a sound and its representation. However, this aspect of the UPIC's operation can also be seen as extending those lessons which may be learned from the purely acoustic into the conceptual domain. For the user, the UPIC provides a sophisticated range of approaches to the nature of sound and graphism. The representation of the sound envelope is a simple analogue; the representation of the waveform is of a different order, requiring the abstraction of physical properties: period and regularity as they relate to harmonicity, smoothness as it relates to harmonic content, relative amounts of deviation of the line as they relate to the amplitudes of component partials, and so on. The wave itself is an abstracted representation of sound, and even quite young children can appreciate this and learn to develop the habit of pattern which influences it, without necessarily supposing that they understand it fully. The concepts involved here, graphism and pattern, the wave, the abstraction of physical quantities, are full and fundamental concepts. The pedagogical approach adopted does not shy away from these, or develop a paternalistic or condescending attitude to their difficulty. It recognises that any approach to these concepts is worthwhile and helps to form a type of competence and understanding quite different to that obtained, say, by learning the pragmatic disposition of knobs and buttons on a keyboard.

In fact other systems for the synthesis of sound don't fare much better in this area. The parameters of the FM algorithm, for example, are notoriously opaque, and many pedagogical approaches just avoid these issues completely to concentrate on more expressive matters. The feeling that the user is actually creating the details of a sound, and the fact that a gradual understanding emerges from the initially ungoverned flow of the hand, engage the user and impart a type of understanding more or less dissociated from the presence of the UPIC depending on the capacity of the individual.

To the more advanced student, the drawing of waveforms also presents a few problems. It is almost impossible to drawn smooth waveforms accurately with a pen (completely impossible with a mouse!), and it requires some experience to predict with much accuracy the sonic outcome of a given shape. The UPIC's sampling capability can again provide a useful resource here, both by producing actual, usable waveforms, and by giving models to investigate and imitate (figure 3).

However, if one simply construes all these 'problems' as benefits, one begins better to understand the pedagogical thrust behind the UPIC's sparseness. There is an intentionality here. There are no easy, preset options. The student is, from the start, brought up short against the very nature of sound and its perception. The challenge of creating musical output must be tackled head on and with a creativity intimately allied with the very nature of the UPIC itself. There is no 'virtual orchestra' here: the imagination must seize on the possibilities available in the system, whose strengths are a direct access to the intimate details of sound, extreme simplicity of means and great speed of working. In my experience, people find this invigorating rather than forbidding.

There is a third, perhaps less obvious, issue which is the relationship between the perceptual unity of a sound and the possible multiplicity of its graphic. There is a strong tendency, even among those familiar with electroacoustic music, to expect a single waveform/envelope complex - or arc - to do too much on its own. The final stage of working on the UPIC, once a satisfactory palette of waveforms and envelopes has been drawn and stored, is to develop a graphic score of some music, where pitch is represented on the vertical axis and time is represented on the horizontal axis (figure 4).

Now the issue of timbre, so-called, becomes crucial, and at the same time an inadequate term for what is required. Perhaps because of the dominance of the notion of the instrument in music, beginners on the UPIC often spend an inordinate amount of time trying to draw 'interesting' waveforms and envelopes rather than learning to synthesise these by using superimpositions of arcs with different waveforms and envelopes. The UPIC is capable of reproducing 1,024 arbitrary waveforms at any one moment, which makes it an extraordinarily powerful additive synthesis tool. This allows the user to think inside the detail of a sound and to draw out the various components as separate arcs which coalesce into one sound mass, or into opposing sound masses. Because of the speed of the real-time system, one can hear the result the moment the arcs have been drawn, and this fast feedback cycle allows the user to learn quickly a number of crucial acoustic lessons: the way the amplitude of a component sound allows it to coalesce or separate out, the way envelopes for different aspects of a sound have to integrate in time, and so on. Thus a particular way of drawing emerges, which perhaps begins with quite primitive, unitary graphic images and then develops a density and detail intimately related to the internal life of the created sounds. The hand and the ear start to form a natural alliance where the eye had dominated previously. This is a process of learning which is uniquely associated with the structure of the UPIC system.

In the course of several years working closely with les Ateliers UPIC, and its then director, Alain Despres, I assisted in the presentation of many education projects and workshops, of the most varied 
The UPIC system

39

FOFME [' OHLE

3

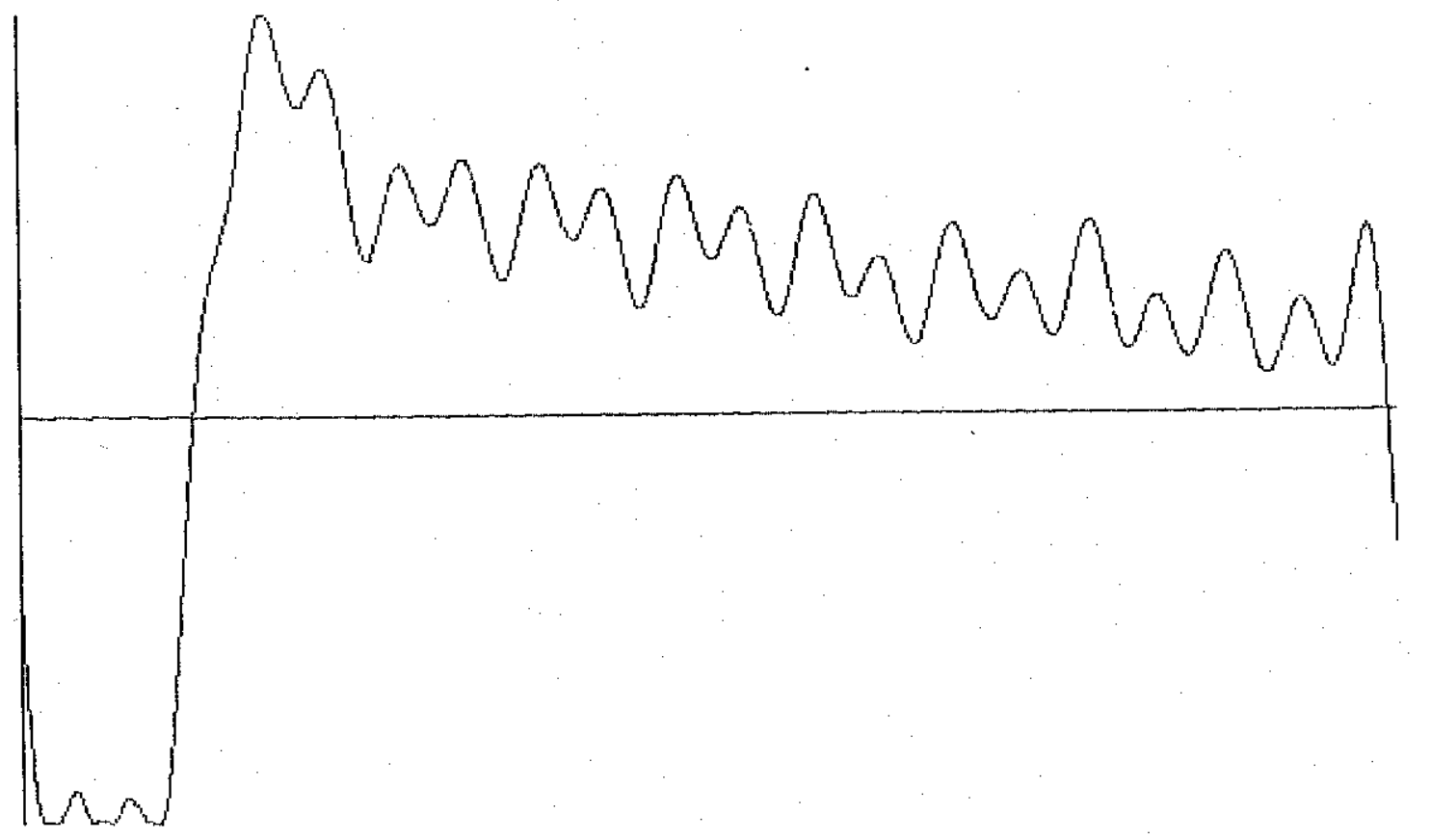

Figure 3

F'FEE

6

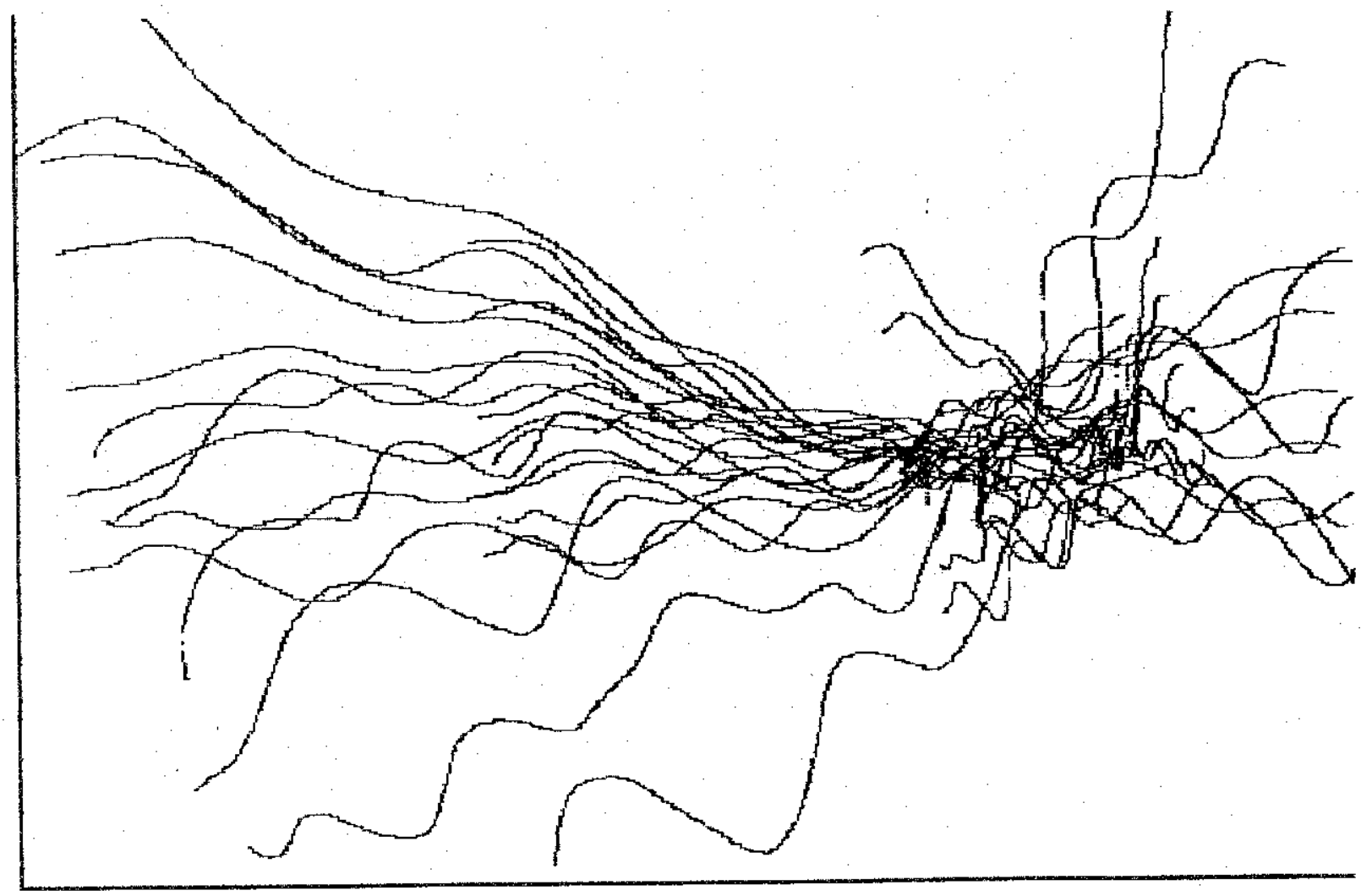

Figure 4 
sort: at the Huddersfield Festival and the Barbican Centre in England, at the Banff Centre, the Montreal Conservatoire and in Toronto, Canada, at the 1990 ICMC in Glasgow, and at the Atelier itself in Paris. Except in Paris, where the courses were run exclusively for composers, the projects usually followed the same basic scheme: group workshops for young people aged between ten and eighteen in the mornings and early afternoons, workshops for a small group of composers in the afternoons and evenings, public demonstrations between workshops, and, in the case of the Glasgow ICMC, sessions for groups of young people with special needs. Thus the systems were always busy and there was a certain premium placed on the number of people who could be involved. Although group working is completely possible on the large tables, and indeed encouraged in the early stages of work, later on individual time is required to accomplish much. On the other hand, once the basics are mastered, a lot may be accomplished speedily. The composer, Richard Barrett, for example, completed a substantial and original work in the few moments of 'down time' during two weeks at the Barbican Centre in London. This point merely demonstrates the speed and ease with which the basics of the system may be learned. The difficulties are all conceptual and creative, not operational.

This brings me to a consideration of some of the main features of the organisation of the workshops. The workshops for school-age children would normally run for one and a half to two hours every day during a week, with a concert at the end including all the work done by the parallel workshops, alongside pieces by Xenakis and other composers who had completed works on the system.

For a class of, say, 24 students, the first session would be a large group session with the aim of introducing everyone to the layout of the system and the concepts of envelope, waveform and frequency. A short, spoken introduction, with appropriate demonstrations on the UPIC, would be followed by the class splitting into two (there were usually two systems in the studio) in order to give each participant a chance to verify those ideas for themselves. The facilitator would simply instruct each participant in what to do with the pen - press here, draw there - with the rest of the group watching, and after the first few members of the group had proceeded in this way the group itself was enouraged to take over this supervisory role. The basic moves are so simple that, even though (or maybe even because!) the table commands are labelled in French, this first session would result in everyone mastering them and being able to recite the command chains.

The emphasis throughout this process was not on the pragmatics but on the principles underlying the production of sound: loudness, frequency, and the way these can vary over time. Despite frequent requests, no attempts were ever made on the part of the facilitators to produce electric guitar sounds or barking dogs. It was always part of the ethos of the workshop that the sounds which resulted were strange and unfamiliar, and that they had to be listened into in order to find their life and meaning. The imaginative process was thus centred on the development of graphic elements and the structural ways in which these related to the perceived sounds, rather than on any 'sounds-like' analogies. 'What happens if ...?' turned out to be a more useful question. At this point, the end of the first session, most participants were able to work unaided, and a moment or two would be left for each group to fend for itself.

After this initial session, the unwieldiness of the twelve-or-so-person group was too much for the situation. There are various solutions to this problem, but the best seemed to be to allow the participants back on subsequent days in groups of three or four, so that everyone had had a chance of individual work by the time of the final concert. This made necessary some further input from the class teacher away from the studio in order to focus the project sufficiently to produce a final piece of work. Thus each group would record the morning's work and take the cassette back to school for comments and planning by the whole class. Sometimes groups would even arrive with predrawn graphics for pages of score, although these did not always survive transfer to the machine. Workshop sessions with these small groups would consist of the groups working largely unaided, with the facilitator on hand to help with technical problems and to stimulate discussion on muscial matters.

Figure 5, for example, shows a page of score developed by pupils from Helme Church of England School, Kirklees. Several graphic motifs and their elaborations are clearly evident here: the starburst patterns, varied in size and density, which are obviously a compromise between a visual inspiration and the feedback from hearing the resulting sounds; the high, repeated sound object at the start of the page, with its more large scale consequent, and a final presentation, low and extended in time at the end of the page; the sine wave motif, beginning singly and developing a parallel motion; the scatters of small lines which in fact develop a more melodic, noteevent material; and so on. The basic materials are limited, and a clear structure is developed combining purely graphic and sonorous considerations.

In the end, besides a piece of music that is clearly a communal effort, there are always significant conceptual gains. Almost all participants gained some appreciable understanding of acoustic concepts, and even such discoveries as why you cannot draw a circle in an area where one of the axes is time are valuable 


\section{PHGE 63}

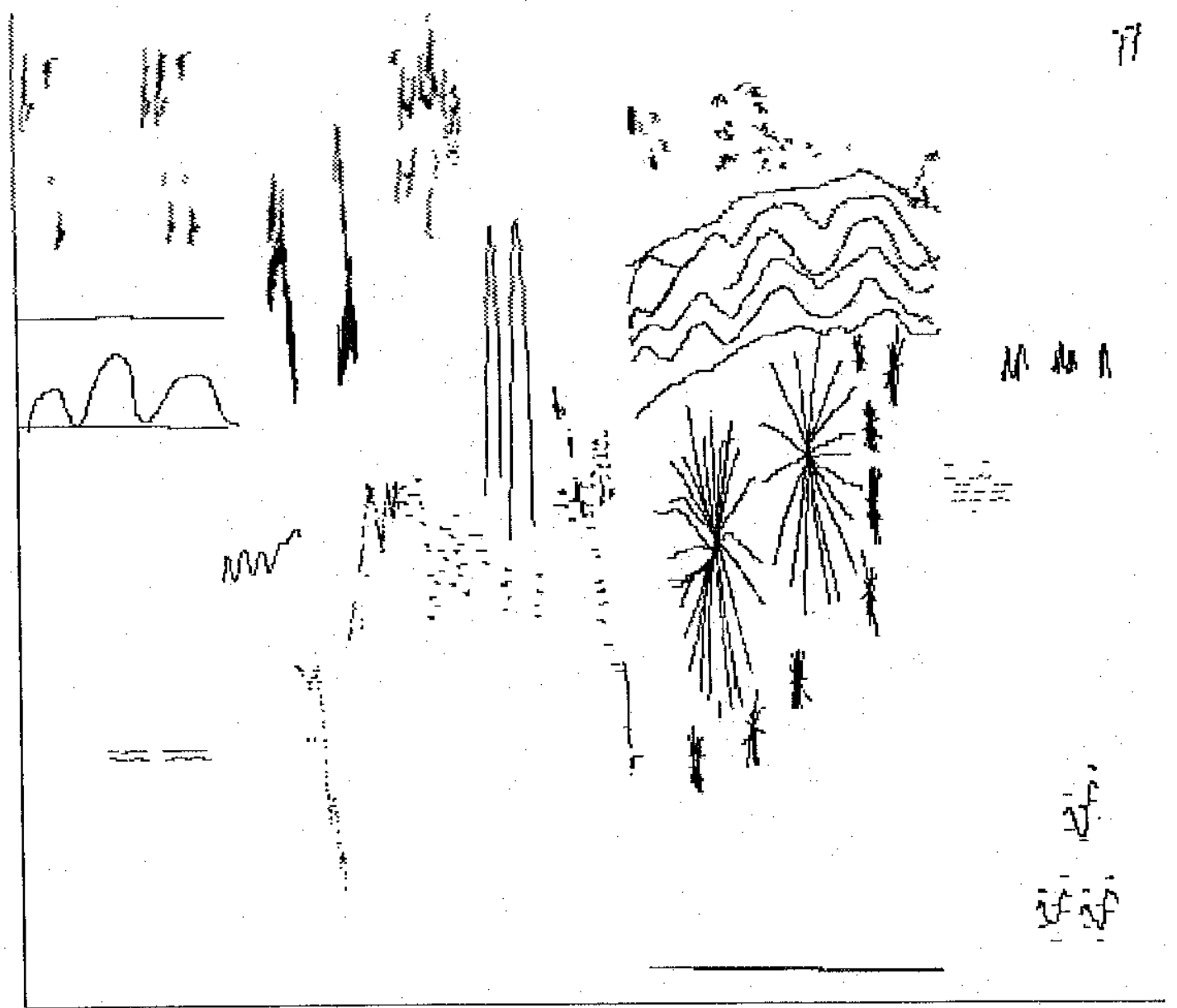

Figure 5

and illuminating. The consequent, accidental rediscovery of the sine wave is an event with ramifications outside the music room. Notions of structure in time are graspable in a very immediate way through the presence of patterns and the developments of shapes and textures. The older school-age participants sometimes found the lack of a familiar sound world disconcerting, but even the UPIC can slip into its own clichés! One group who attended the Barbican Centre workshops, for example, took the consequences of drawing note events head on and produced some very convincing reggae. In this way the main outcome of working with the UPIC is the development of the musical and the conceptual imagination, unhampered by an onerous technical expertise.

The particular nature of the UPIC interface has also made possible workshops involving those with special needs. At the ICMC in Glasgow in 1990, for example, a number of special needs groups, including a dance ensemble, made use of the system and its capacity for physical reconfiguration. In particular the scale of the drawing surface can be varied to allow for varying capabilities of movement and the control points of contact can be enlarged and repositioned to the needs of the user. This allows almost a normal use of the system whatever the user's physical capabilities. In every other respect the workshops could be presented as normal, and the dance ensemble, for example, created and performed a dance piece which worked by the careful coordination of concepts of movement and graphics. Where the normal conceptual structure of the workshop was not appropriate, the development of a more expressive approach to graphic development was still possible, while the facilitator could take over the task of operating the control functions of the system. In these situations group working was usually still possible, though each participant would be allowed a significant portion of time alone at the table, with the others listening and observing, and with a consequent increase in the number of facilitators involved. Here the disconnection in time between drawing and hearing back the sound, however small, is a significant problem. This involves the development of a facilitating technique 


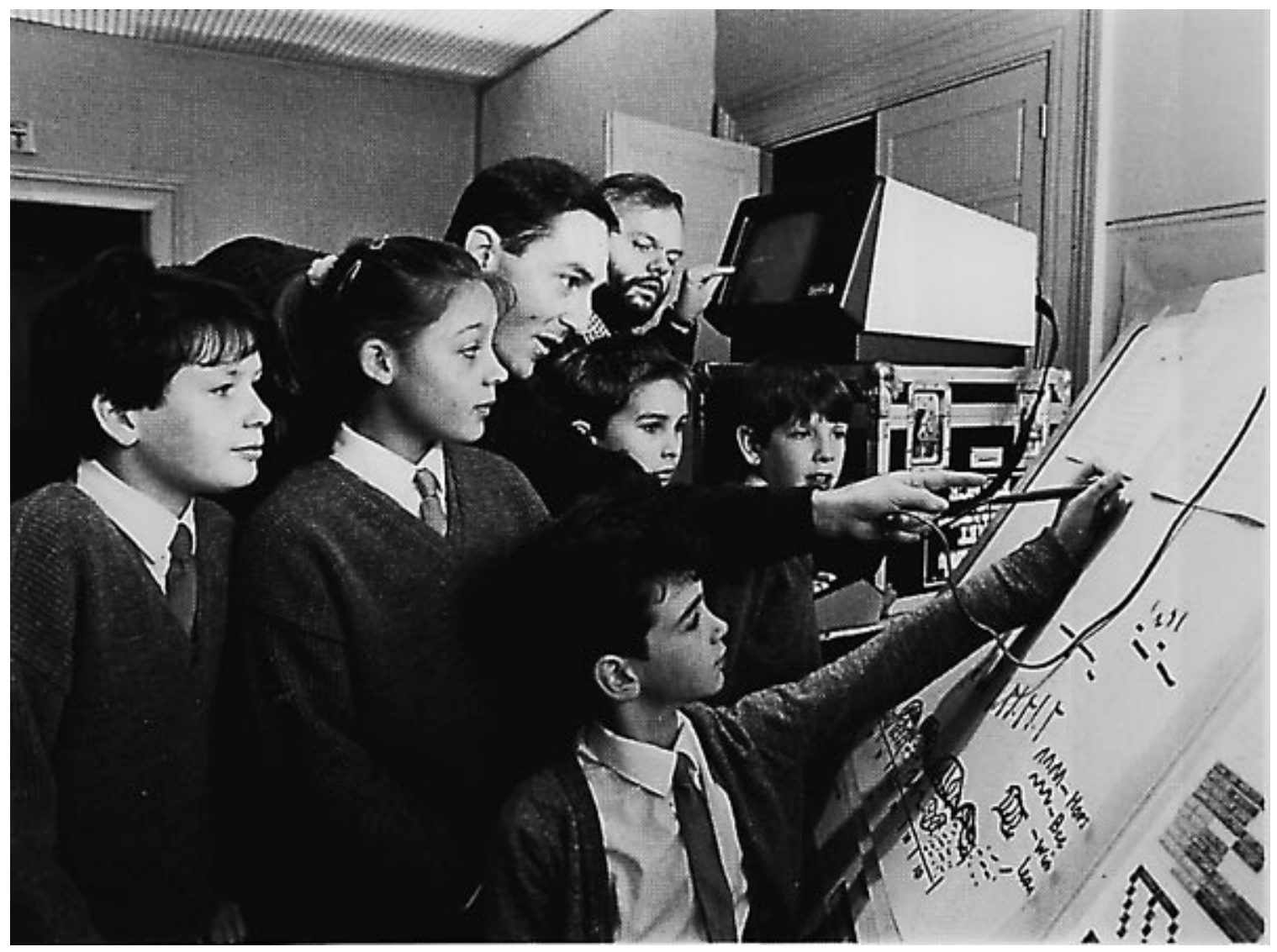

Figure 6. School pupils using the UPIC system (by courtesy of the Huddersfield Daily Examiner).

which effectively separates the drawing and listening situations, or which makes use of the ability of the system to play single timbres in real-time improvisation without the storing of the arcs. The output could simply be recorded by microphone in this instance.

Finally one could say that the UPIC is also a system for artistic creation by composers and even by graphic designers. Yet the system used by Xenakis, and at this point by a hundred or so other composers, is exactly the same as that used by every workshop participant. Each participant may hear Mycenae Alpha of Xenakis knowing that they have mastered the same concepts and controls as were used to produce that piece, and that their own work stands in direct relation to it at the musical level. And this is a crucial aspect of the pedagogical attitude embodied in the UPIC; that everyone is musical, and the proper meeting place is the plane of concepts and the imagination.

\section{REFERENCES}

Lohner, H. 1986a. The UPIC system: a user's report. Computer Music Journal 10(4). Cambridge, MA: MIT Press.
Lohner, H. 1986b. Interview with Iannis Xenakis. Computer Music Journal 10(4). Cambridge, MA: MIT Press.

Marino, G., Razcinski, J.-M., and Serra, M.-H. 1990. The new UPIC system. In S. Arnold and G. Hair (eds.) Proc. ICMC. San Francisco: CMA.

Marino, G., Razcinski, J.-M., and Serra, M.-H. 1991. New UPIC system demonstration. In B. Alphonce and B. Pennycook (eds.) Proc. ICMC. San Francisco: CMA.

Marino, G., Razcinski, J.-M., and Serra, M.-H. 1993. The UPIC system: origins and innovations. Perspectives of New Music XXXI(1).

Matossian, N. 1986. Xenakis. London: Kahn \& Averill.

Mosch, U. 1993. Klangbild-Bildklang: Das UPIC-Gerät von Iannis Xenakis. Positionen: Beiträge zur neuen Musik 17.

Powell, C. 1988. L'UPIC du CENAMu (sic): a Report of a Course using Xenakis's UPIC at les Ateliers UPIC in Paris, 22 June to 10 July 1987. Canzona IX/29.

Xenakis, I. 1992. Formalised Music, Thought and Mathematics in Composition, revised edn. Stuyvesant, NY: Pendragon Press. 